\title{
Visual body size adaptation and estimation of tactile distance
}

Regine Zopf $f^{1,2,4}$, Veronika Kosourkhina ${ }^{3}$, Kevin Brooks ${ }^{2,3,4}$, Vince Polito ${ }^{1,4}$, lan Stephen ${ }^{2,3,4}$

1. Department of Cognitive Science, Faculty of Medical, Health \& Human Sciences, Macquarie University, Sydney, Australia

2. Perception in Action Research Centre, Faculty of Medical, Health \& Human Sciences, Macquarie University, Sydney, Australia

3. Department of Psychology, Faculty of Medical, Health \& Human Sciences, Macquarie University, Sydney, Australia

4. Body Image and Ingestion Group, Department of Psychology, Macquarie University, Sydney Australia 


\section{Abstract}

Estimating the size of bodies is crucial for interactions with physical and social environments. Body size perception is malleable and can be altered using visual adaptation paradigms. However, it is unclear whether such visual adaptation effects also transfer to other modalities and influence, for example, the perception of tactile distances. In this study we employed a visual adaptation paradigm. Participants were exposed to images of expanded or contracted versions of self- or other-identity bodies. Before and after this adaptation they were asked to manipulate the width of body images to appear as "normal" as possible. We replicated an effect of visual adaptation, such that the body size selected as most "normal" was larger after exposure to expanded and thinner after exposure to contracted adaptation stimuli. In contrast, we did not find evidence that this adaptation effect transfers to distance estimates for paired tactile stimuli delivered to the abdomen. A Bayesian analysis showed that our data provide moderate evidence that there is no effect of visual body size adaptation on the estimation of spatial parameters in a tactile task. This suggests that visual body size adaptation effects do not transfer to somatosensory body size representations.

Keywords: body perception; visual adaptation; tactile distance; body misperception; size distortions 


\section{Introduction}

A crucial aspect of how we interact with the world and also evaluate our own self is the perception of our own and others' bodies. In particular, spatial parameters such as body size are important for how we place ourselves relative to the environment, including our socially relevant peers. To optimise our interactions with the world around us, our perceptual systems have evolved mechanisms to adjust to structural and physiological changes in our bodies and sensory organs, for example due to developmental changes, as well as adjust to changes in our environmental context, for example when moving from the city to the sea. These mechanisms include the ability to adjust to statistical regularities in sensory input through perceptual learning and adaptation. Following this principle, perception of body size seems to be highly dynamic and adjustable.

For example, a number of studies have demonstrated adaptation to visually perceived body size (see Brooks, Mond, et al., 2019; Challinor et al., 2017 for reviews). Prolonged exposure to contracted (or expanded) bodies results in adaptation after-effects such that subsequently-presented bodies are perceived as larger (or smaller) than they really are. This effect has been shown to affect high-level visual body representations and does not appear to be merely a collection of low-level adaptation effects (Brooks, Clifford, Stevenson, Mond, \& Stephen, 2018). Furthermore, visual adaptation can affect representations of different structural body components, such as body fat and body muscle, independently (Sturman, Stephen, Mond, Stevenson, \& Brooks, 2017). Adaptation effects have also been shown to transfer between identities (Hummel, Rudolf, Untch, Grabhorn, \& Mohr, 2012). However, there seems to be an identity-specific component such that adaptation effects are larger when the identity of adaptation and test stimuli is congruent (Brooks, Mond, Stevenson, \& 
Stephen, 2016). Overall these experimental findings demonstrate that high-level visual body size representations adapt to recent visual input and can be quite rapidly modified.

A representation of one's bodily shape and size is also important for the perception of somatosensory information (Longo, Azanon, \& Haggard, 2010; Medina \& Coslett, 2010; Gadsby, 2017). This is, for example, important for tactile recognition and interaction with objects where one has to estimate the distance between points that are touched on the skin. The spatial relationships between different touches on the skin cannot be simply determined by activation in the primary somatosensory cortex because it contains distorted representations, known as the somatosensory homunculus (e.g., larger representations for more sensitive skin regions; (e.g., larger representation for more sensitive skin regions; Penfield \& Boldrey, 1937). Thus, for perceptual estimates of spatial distance between two touches on the skin, a model of body size and shape and a transfer function is needed to map signals from the primary somatosensory cortex to a more veridical spatial representation of the body. The generation of a model of body size and shape is thought to involve sensory integration of information from different modalities including somatosensory with visual input (Longo, 2015).

Previous research has indeed shown that short-term manipulations of visual, proprioceptive and auditory input can rescale the body size model and change tactile distance and haptic size estimations (Taylor-Clarke, Jacobsen, \& Haggard, 2004; Bruno \& Bertamini, 2010; de Vignemont, Ehrsson, \& Haggard, 2005; Tajadura-Jimenez et al., 2012). For example, TaylorClarke et al. (2004) have shown that altering the visual experience of the body and viewing an enlarged arm or hand increases tactile distance estimates. The authors propose (p.220) "[...] that rescaling may be particularly important for touch, because primary somatosensory 
cortical representation is highly plastic, varying with tactile experience and bodily changes." This demonstrates that prior visual experience can affect somatosensory spatial representations.

However, to date it is unclear whether visual size adaptation demonstrated for whole bodies also rescales the perception of tactile distance. It is possible that viewing a contracted or expanded whole body for some time changes both the visual representation of body size, and also transfers to size representations used for the perception of tactile distances on the abdomen. For example, after adaptation to contracted (or expanded) bodies, images of bodies are subsequently visually perceived as larger (or smaller). This might also affect an internal mental representation of one's own body and lead to increased (or decreased) estimates of tactile distances after contracted (or expanded) body size adaptation. Alternatively, visual exposure to contracted (or expanded) bodies might also lead to a subsequent decrease (or increase) of the perceived distance between two tactile stimuli, especially if the participant internalises the visual stimulus as an accurate representation of their own body, as occurred for arm and hand stimuli in a previous study (Taylor-Clarke et al., 2004). Or, it may be that simply viewing small images of bodies from a second person perspective causes visual body size adaptation, but has no effect on the multi-modal size representation of one's own body. If this were the case, judgements of tactile distance would remain unchanged.

In this study we set out to investigate whether body size modulations might transfer across different modalities and tasks. This issue has theoretical relevance for understanding how body size is represented in the human brain, and for understanding what mechanisms might be able to explain body size misperceptions. An overestimation of body size and shape, in 
particular for the middle third of the body, is a core aspect of body image disturbance in eating disorders (Bruch, 1962). Visual adaptation has been hypothesised as a potential mechanism for body-size and -shape misperception (Brooks, Mond, et al., 2019; Challinor et al., 2017; Mohr, Rickmeyer, Hummel, Ernst, \& Grabhorn, 2016; Winkler \& Rhodes, 2005). The idea is that long-term exposure to extreme body images, for example in the media, leads to visual aftereffects causing all images of bodies to appear larger than they really are, including one's own body (Brooks, Mond, et al., 2019). In line with this hypothesis, Mohr et al. (2016) have reported a reduced capacity to adapt to contracted thin bodies in individuals with eating disorders (anorexia and bulimia nervosa). The authors suggest that after longterm adaptation to thin body shapes during the participants' real-world experiences, additional visual adaption to thin body shapes during laboratory sessions might not be possible. Although relevant alternative explanations such as response biases could not be ruled out by the authors, this may suggest long-term changes to the body size representation, possibly due to disproportionate viewing of thin bodies over time. In somatosensory tasks, enlarged spatial representations have also been linked to anorexia nervosa. Several studies have shown that tactile distances were overestimated, especially for the abdomen and the horizontal/width dimension, in a group of individuals with anorexia nervosa as compared to controls (Keizer et al., 2011; Keizer, Smeets, Dijkerman, van Elburg, \& Postma, 2012; Spitoni et al., 2015). This supports the possibility that a representation that tracks spatial body dimensions might be impaired in anorexia nervosa, which in turn could affect a number of different body representations and tasks relying on an estimate of body size (Gadsby, 2017). On the basis of the hypothesis that visual adaptation might be a causal mechanism for body size misperceptions in eating disorders 
(Brooks, Mond, et al., 2019), it is thus important to test whether visual adaptation effects could also lead to changes in tactile distance estimation.

\section{Methods}

We employed a visual body size adaptation paradigm similar to our previous research (Brooks, Baldry, et al., 2019; Gould-Fensom et al., 2019) and tested the effect of body size adaptation on the visual perception of body size as well as tactile distance.

\section{Participants}

Sixty female Caucasian students (age range from 18 to 30 years, $M=20.25, S D=3.2$ ) enrolled in an undergraduate Psychology course at Macquarie University received course credit for their participation. All participants had normal or corrected to normal vision and gave written consent before agreeing to participate. The experiment was approved by the Macquarie University Human Research Ethics Committee and conducted in accordance with the declaration of Helsinki.

\section{Design}

In a $2 \times 2$ factorial between-subject design we manipulated adaptation direction (contracted versus expanded) and adaptation identity (self versus other). We tested 15 participants for each of the four conditions and measured two dependent variables: a visual point of subjective normality (PSN: the visual stimulus size that appeared most normal) and a tactile distance estimation (TDE: an estimate of the distance between two tactile stimuli presented on the participant's abdomen). We measured both variables before (baseline) and after an adaptation phase. 


\section{Apparatus and Stimuli}

We presented the visual stimuli on an AOC monitor (G2770PF) with a screen resolution of $1,920 \times 1,080$ pixels. Participants were standing for optimal stimulus delivery in the tactile task (see below) and the monitor was placed on top of a raised platform (a heightadjustable standing desk) to be at the participant's viewing height. The viewing distance was approximately $80 \mathrm{~cm}$.

We created full body images, including the face, from digital photographs of each participant. In those images participants wore grey cycling shorts and a grey singlet to ensure good visibility of the body shape. Participants removed jewellery and make-up before their pictures were taken. We asked participants to pose with a neutral expression in a standard anatomical position (standing upright, feet positioned approximately at shoulder width, arms straight at the side of the body, hands $\sim 20 \mathrm{~cm}$ from the body with palms facing forward, see Figure $1 \mathrm{~A}, \mathrm{~B})$. We took the images in a booth painted with Munsell N5 neutral grey. The booth was illuminated with 15 high-accuracy d65 fluorescent Philips tubes mounted in high-frequency fixtures to reduce the effects of flickering. The camera (Canon EOS 70D) settings were held constant across all participants.

We measured weight and height to obtain a measurement of the Body Mass Index (mean $\mathrm{BMI}=22.85, \mathrm{SD}=3.41)$. Participants in "other" conditions were assigned a body for the adaptation phase (the visual testing stimuli were always self-images) from an existing database of images collected under identical conditions. The "other" BMIs were matched as closely as possible (within $+/-0.2 \mathrm{~kg} / \mathrm{m}^{2}$ of the participant's BMI). 
To create different body size stimuli we used the "spherize" function in Adobe Photoshop to contract and expand the depicted bodies from neck to ankles with the head size and importantly also the arm/hand size unchanged. To avoid image discontinuities we used a feathered marquee which blended the body distortions smoothly into the non-manipulated areas of the image. We created thirteen test images involving spherize manipulations of between $-30 \%$ and $+30 \%$ in $5 \%$ steps, including the original image $(0 \%)$. The bodies were formatted to a standard height of 720 pixels and a width of 320 pixels (total image size $900 \mathrm{x}$ 450 pixels). We used the most extreme images (-30\% and $30 \%)$ as adaptation stimuli (Figure $1 \mathrm{~A})$. Examples for test stimuli at $+/-20 \%,+/-10 \%$ and $0 \%$ spherize levels are depicted in Figure 1 B (Brooks et al., 2016).

For tactile stimulation we used four small electromagnetic solenoid-type stimulators (diameter: $18 \mathrm{~mm}$ and probe height: $12 \mathrm{~mm}$, Dancer Design, St. Helens, UK; dancerdesign.co.uk) and an amplifier (TactAmp 4.2 with a D25 serial port, Dancer Design). Small solenoid stimulators have also been used in previous tactile distance estimation tasks (de Vignemont et al., 2005; Taylor-Clarke et al., 2004). We attached the tactile stimulators in a row to a cloth belt (with 2, 5 and $2 \mathrm{~cm}$ between the tactors' probes; this allowed the delivery of tactile stimuli with 5, 7 and $9 \mathrm{~cm}$ distances; Figure $1 \mathrm{C})$. The tactile stimuli consisted of a 1.2 second vibration $(33 \mathrm{~Hz})$. We placed two further "sham tactors" that were not connected to the amplifier on both the outer left and the outer right side with $2 \mathrm{~cm}$ spacings. This way participants were not able to guess tactile distances from just feeling the location of the tactors alone. We used an electronic vernier caliper (OriginCal, iGaging, California, USA) connected via the USB port to record distance estimates for the tactile task (Figure 1D). Two finger attachments for the thumb and index finger were mounted to the 
two caliper sides so that participants could easily move these along using one hand only. To hide their view of the tactile stimulators and caliper, participants wore a black cape and the bottom of the cape was attached to the table.

We used Matlab (MathWorks, Natick, Massachusetts, U.S.A.) and the Psychophysics Toolbox (Brainard, 1997; Kleiner et al., 2007) to control stimulus delivery and response collection. Participants used their right hand for tactile caliper responses and the left hand to control stimulus presentation and give responses for the visual task. 
B

A Examples of Adaptation Stimuli
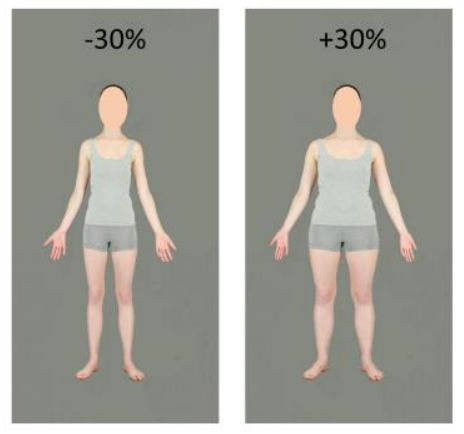

C
Examples of Visual Test Stimuli

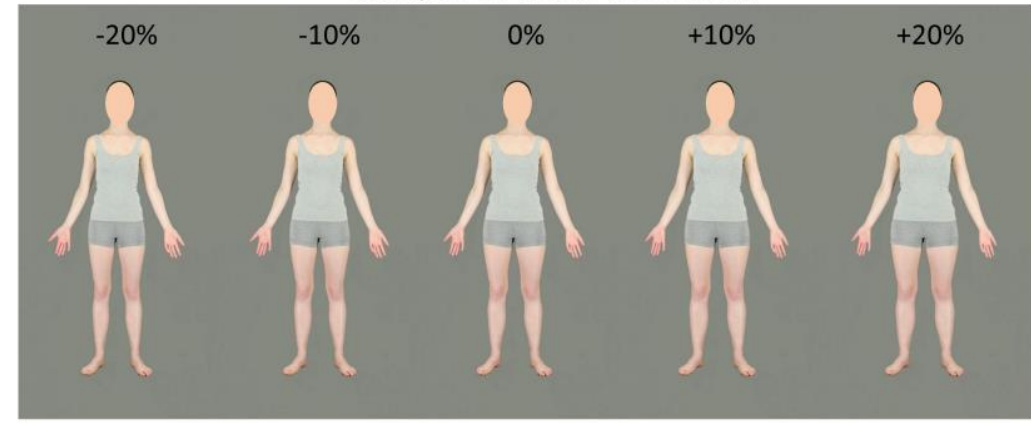
Schematic of Tactile Test Stimuli

D Tactile Response Caliper
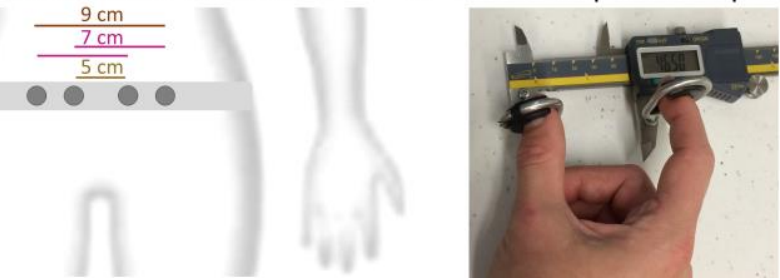

Figure 1. Methods. A) Examples of adaptation stimuli for the contraction condition (-30\%)

and the expansion (+ 30\%) conditions. B) Five examples for the visual test stimuli at $-20,-10$, $0,10,20 \%$ size manipulation levels are shown. Faces were fully visible in the experiment. D) Schematic of the location and distances used for tactile stimulation. Participants wore a belt with four tactile stimulators centred just below the navel with paired distances of 5, 7 and 9 $\mathrm{cm}$ in between. C) Picture of caliper used to provide tactile distance estimate. 


\section{Tasks}

To measure the visual point of subjective normality (PSN) we used a method of adjustment task (Brooks, Baldry, et al., 2019; Gould-Fensom et al., 2019). In each visual response trial, participants moved the mouse horizontally (starting position was randomised, mouse pointer was not visible) to move through the 13 possible body images (see 1B). The task was to select the image that looked most normal, pressing a mouse button to register their response.

For the tactile task, we used a tactile distance estimation task. Following tactile stimulation on the abdomen, participants set the distance with the calipers that they perceived to match the abdominal stimulus distance. This is analogous to holding one's fingers apart to say "they are this far apart". Once they completed their distance estimate, participants pressed a button on a small box to enter their response setting. After a one-second interval participants were then instructed to either completely close or widen the caliper as far as they could. They then were asked to press the mouse button which initiated the next trial. The type of instruction (close versus widen) and the start position for the next trial was randomised. There were three types of tactile trials with stimuli either 5, 7 or $9 \mathrm{~cm}$ apart. In half the trials the $7 \mathrm{~cm}$ distance was achieved using the outer left and middle right tactile stimulator and in the other half using the outer right and middle left tactile stimulator.

Visual and tactile trials were presented in a pseudo-random order in randomly generated sets of four trials ( $A D B C, C B D A, A C B D$, etc.) so that each set contained one of each trial type ( 1 visual and each of the 3 tactile tasks; the order of the different $7 \mathrm{~cm}$ trials was randomised within the baseline and adaptation block). A short (50 ms) sound was played 
before each trial (a low tone for the tactile task and a high tone for the visual task) to prepare participants. Each trial started 500 ms after the sound.

\section{Procedure}

The experiment took place 2-7 days after the body pictures were taken. The experiment included equipment setup and a short practice phase to familiarise the participants with the procedure, followed by baseline testing, adaptation and post-adaptation testing. The experiment took approximately 30 minutes to complete.

Participants were standing throughout the experiment. First, the experimenter fitted the belt around the participant's abdomen and centred the tactile stimulators just below the navel. Then participants were shown the unchanged picture of their own body as a reference for five seconds. This was followed by task instructions and the practice phase which included four sets of the visual and tactile test stimuli. This was followed by a baseline block of 10 trial sets, totalling 40 trials.

The baseline block was followed by an adaptation block with 40 trials. The adaptation block started with a 120 second initial adaptation period. During this time the adaptation stimulus (contracted or expanded body of self or other) was shown. Participants were asked to simply view the image which was repositioned on the screen every 3 seconds (up to +/- 30 pixels in all directions from the centre). After the initial adaptation a top-up adaptation period of 18 seconds (refreshed and repositioned as above) was used before each set of four trials. 


\section{Statistical Analysis}

We used R for data processing and visualisation including the package ggplot2 (Wickham, 2016) and JASP (www.jasp-stat.org) for statistical data analysis.

We calculated the relative PSN and TDE change after adaptation compared to the baseline for each condition and participant. To analyse visual PSN and TDE changes, we used $2 \times 2$ between-subject ANOVA with the factors adaptation direction (contracted versus expanded) and adaptation identity (self versus other). The effects of interest to test our hypotheses are the main effect of adaptation direction and the interaction between adaptation direction and adaptation identity. The threshold for statistical significance was set at $p<0.05$.

In addition, to verify that our participants were able to discriminate different tactile distances, we also analysed baseline TDE values using a one-way within-subject ANOVA with the factor tactile distance $(5 \mathrm{~cm}, 7 \mathrm{~cm}, 9 \mathrm{~cm})$.

To further evaluate non-significant results we used a Bayesian analysis (Dienes, 2014). We calculated Bayes Factors (BF) using the JASP default prior (Cauchy prior, $r=0.707$ ). A BF $<1 / 3$ indicates moderate evidence for the null hypothesis (and BF $<1 / 10$ as strong evidence) and $\mathrm{BF}>3$ indicates moderate evidence for the alternative hypothesis (and BF > 10 as strong evidence), whereas BFs between $1 / 3$ and 3 would indicate a lack of sensitivity to support one hypothesis over the other (Jeffreys, 1961; Lee \& Wagenmakers, 2014). 


\section{Results}

As expected we found a main effect of adaptation direction $[F(1,56)=119.77, p<0.001$, $\left.\eta_{p}{ }^{2}=0.681\right]$ with negative values for the contracted condition and positive values for the expanded condition (Figure 2). Furthermore, we found a significant interaction between adaptation direction and adaptation identity $\left[F(1,56)=11.96, p=0.001, \eta_{p}{ }^{2}=0.176\right]$ with larger adaptation effects for self than for other. These results replicate our previous visual body adaptation findings (Brooks et al., 2018; Brooks et al., 2016).

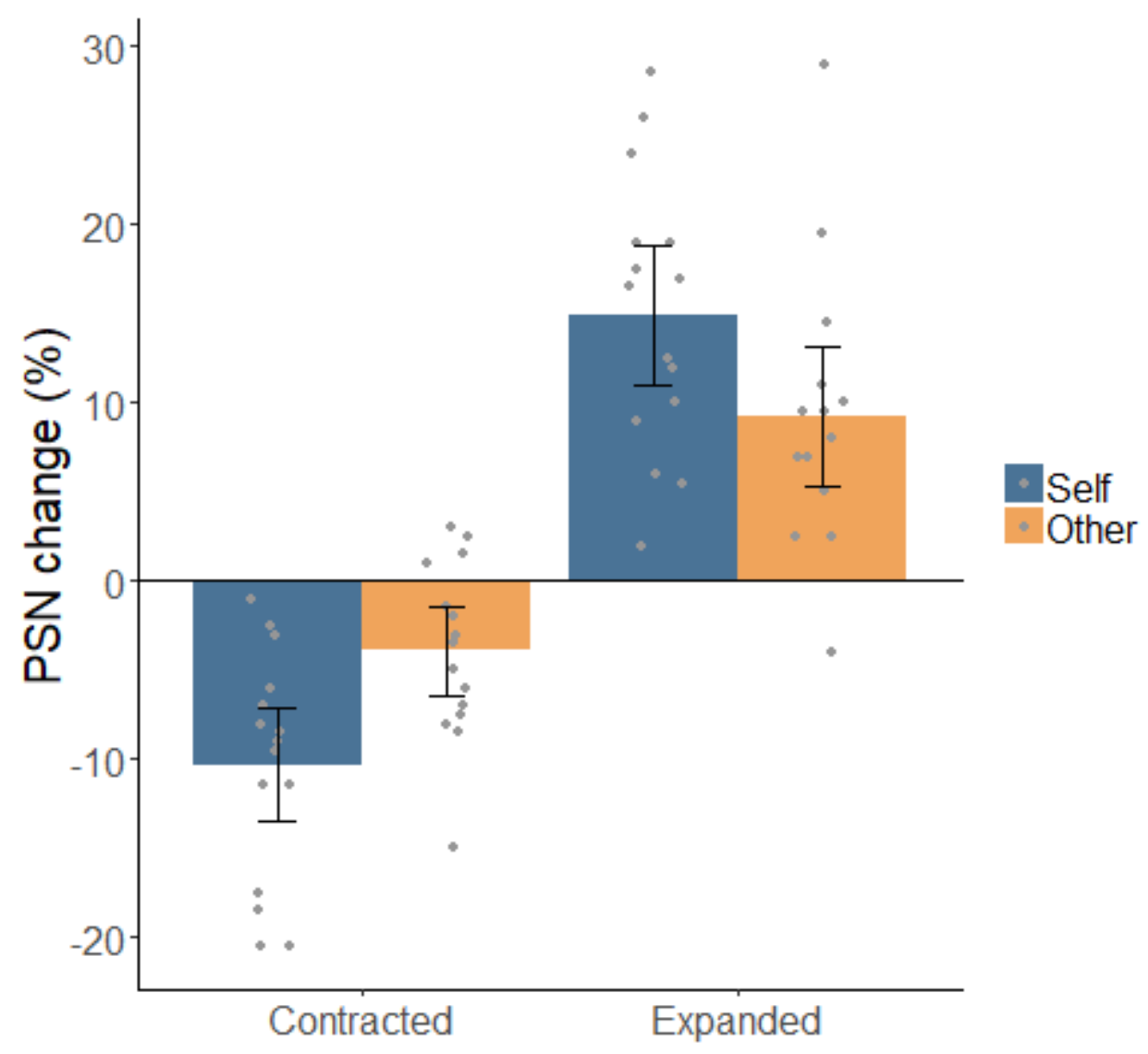

Figure 2. Visual task results. Change of point of subjective normality (PSN) relative to baseline for contracted and expanded body size adaptation conditions for self (blue) and other (yellow) adaptation images. All test stimuli were self-identity. Grey dots represent individual data. Error bars represent 95\% confidence intervals. 
For the baseline TDE values (Figure 3), we found a numerical increase from the $5 \mathrm{~cm}$, to the $7 \mathrm{~cm}$ to the $9 \mathrm{~cm}$ distance, and a significant main effect of distance $[F(2,118)=94.63$, $\left.p<0.001, \eta_{p}^{2}=0.616\right]$. This result shows that participants on average gave distance-specific responses and thus could perceive the distances as different. Across all distances, we observed an underestimation compared to actual distance at each level which has previously also been reported for a control sample (Keizer et al., 2012). As in this previous research, we collapsed the data across the three distances to investigate effects of our manipulations on TDE.

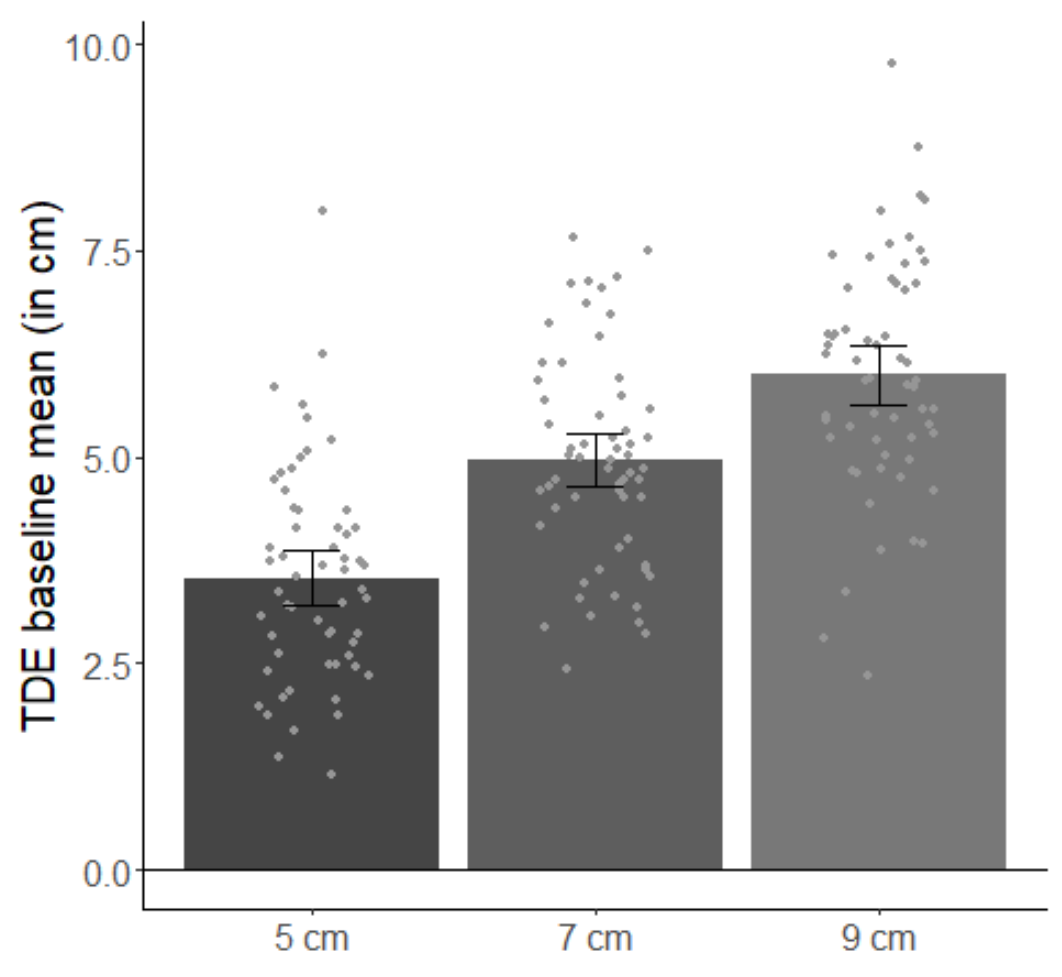

Figure 3. Tactile baseline estimates. Tactile distance estimates (TDE) for the baseline (in $\mathrm{cm})$. This shows estimate differences between the distance conditions and some underestimation of tactile distance. Grey dots represent individual data. Error bars represent 95\% confidence intervals. 
We did not find significant adaptation effects for the tactile task measure (Figure 4). TDE values relative to baseline did not differ significantly between the contracted and expanded condition [main effect adaptation direction: $F(1,56)=0.847, p=0.361, \eta_{p}{ }^{2}=0.015$ ]. Furthermore, we found no significant interaction between adaptation direction and adaptation identity $\left[F(1,56)=0.039, p=0.845, \eta_{p}{ }^{2}=0.001\right] .{ }^{1}$

This result could however also be due to the fact that our methods were not sensitive enough to detect a potential effect. Given that it involves transfer of the effect between two separate sensory modalities, any adaptation effect in the tactile task would likely be smaller than the clearly present adaptation effect in the visual task. Furthermore, the tactile data would be expected to be noisier as humans generally perceive relative distance and size with greater precision in the visual than in the haptic modality (Ernst \& Banks, 2002).

\footnotetext{
${ }^{1}$ In addition and not predicted by previous work, we found a significant main effect for the adaption identity $\left[F(1,56)=4.427, p=0.040, \eta_{p}^{2}=0.073\right]$. Further exploring what may underlie this main effect, we found that both other-groups had smaller baseline mean tactile estimates (thin-other TDE $E_{\text {baseline }}=45.42$, fat-other $\mathrm{TDE}_{\text {baseline }}=44.53$ ) compared to the self-groups (thin-self TDE $E_{\text {baseline }}=50.52$, fat-self TDE $_{\text {baseline }}=52.70$ ). After adaptation, the values for the other-group were larger (thin-other $T_{D E} E_{\text {adapted }}=46.10$, fat-other $T_{D E} E_{\text {adapted }}=46.52$ ) and for the self-group smaller (thin-self TDE adapted $=46.99$, fat-self TDE $E_{\text {adapted }}=51.20$ ) compared to baseline. Thus the groups were more similar in their estimation after adaptation, likely due to regression toward the mean effect (Stigler, 1997) or alternatively because they had more practice with the task. Thus, the group baseline differences, which appear to be an anomaly of random sampling, and which were not present in postadaptation data, may underlie this observed main effect of adaptation identity.
} 


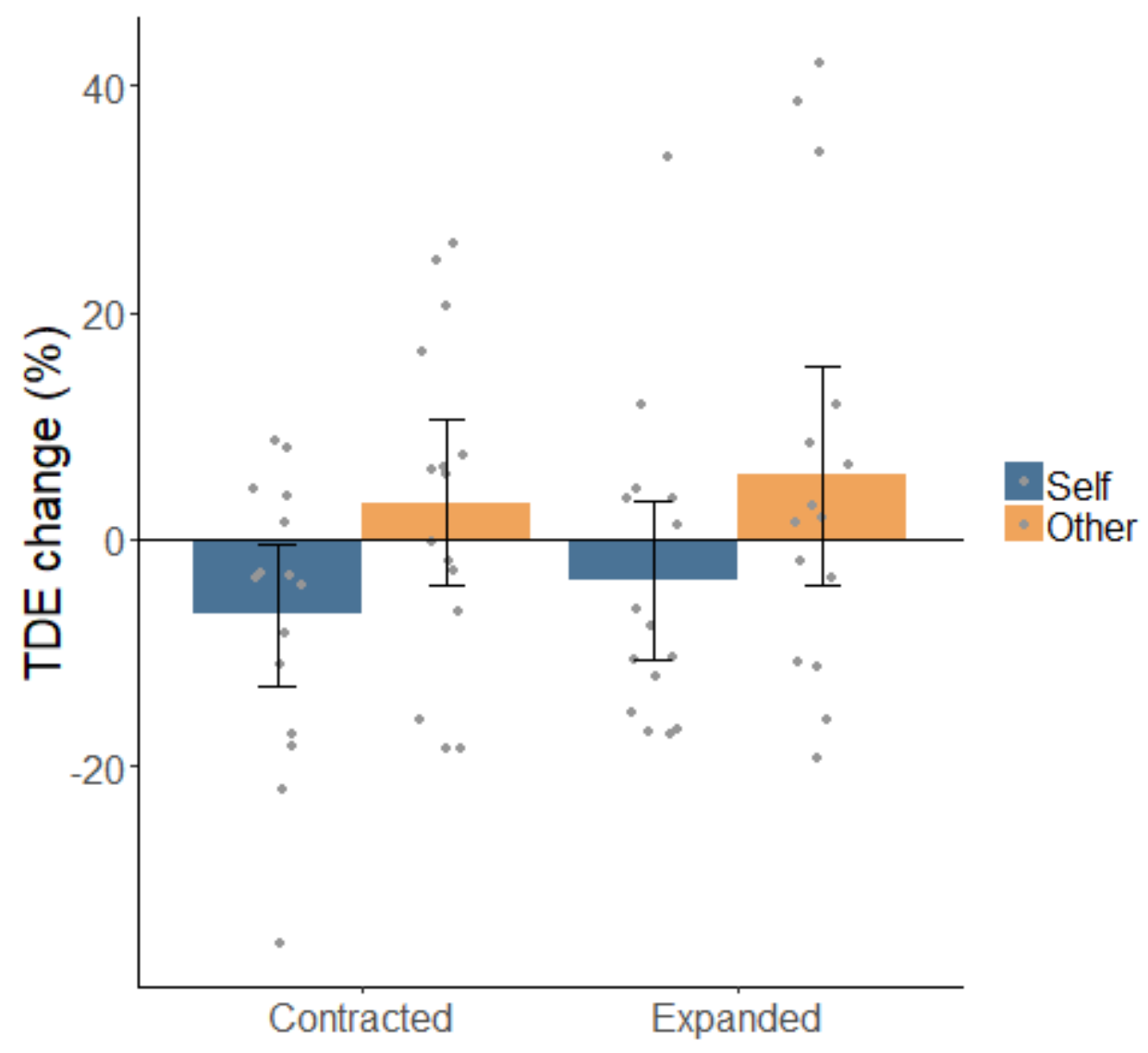

Figure 4. Tactile task results. Change in tactile distance estimation (TDE) relative to baseline for contracted and expanded body size adaptation conditions for self (blue) and other (yellow) adaptation images. Grey dots represent individual data. Error bars represent 95\% confidence intervals. 
To evaluate whether our data were precise enough to provide evidence for the null hypothesis, we combined the data across all groups (Figure 5) and conducted a Bayesian analysis (Dienes, 2014). We combined the data by multiplying all effects that we expected based on visual adaptation to go in the negative direction by -1 (the visual data for the contracted condition and the tactile data for the expanded condition). Thus the hypothesised change due to adaptation effects for both contracted and expanded conditions was subsequently in the positive direction.

As expected for the visual data we found strong evidence for an adaptation effect ( $B F=7.73$ $\left.\times 10^{10}\right)$. However, for the tactile data we found moderate evidence for the null hypothesis $(B F=0.209)$. This provides support for the idea that visual body size adaptation does not influence tactile distance estimations on the abdomen under our experimental conditions. 


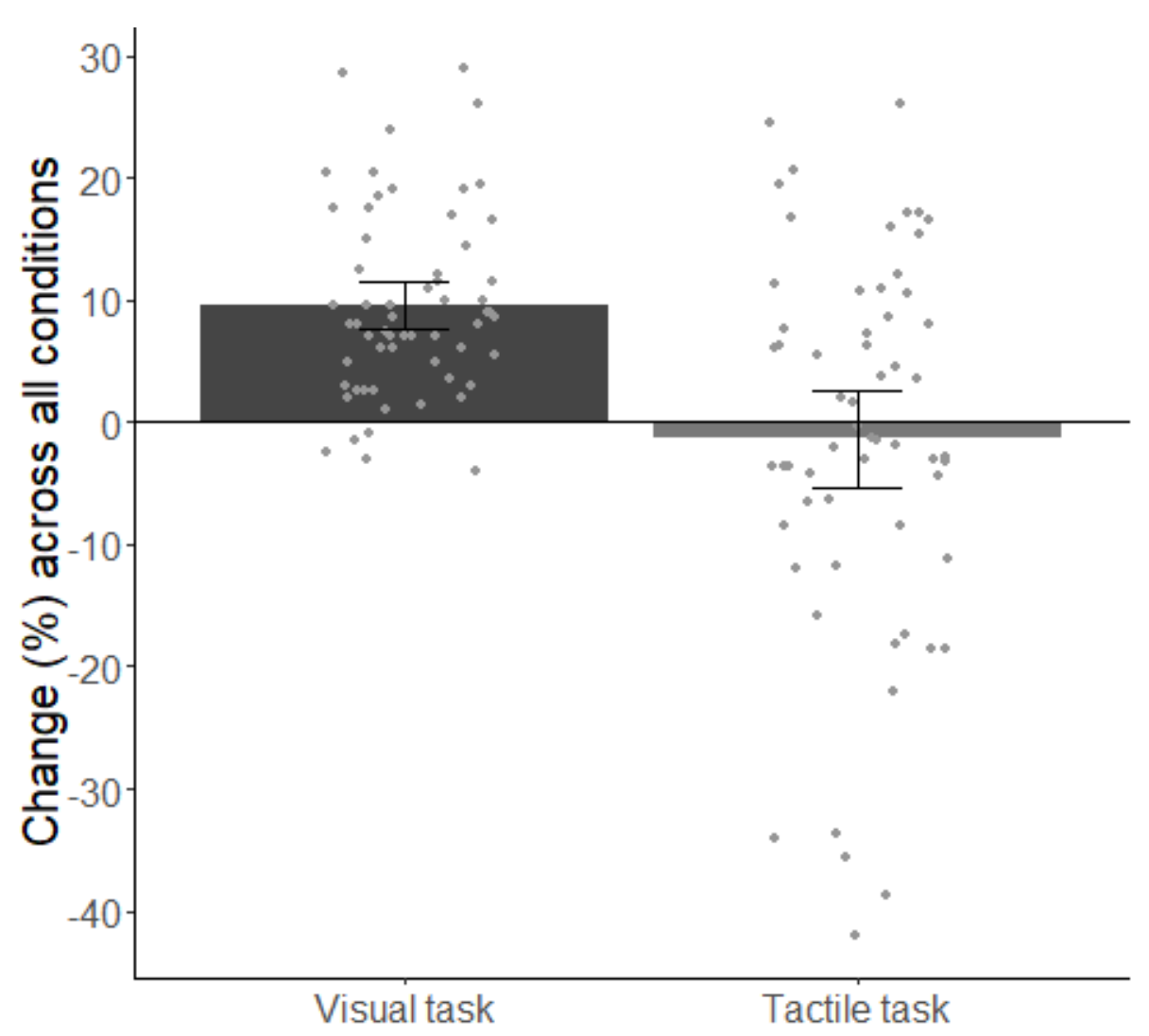

Figure 5. Visual and tactile results combined across conditions and replotted. We combined the data (expected direction positive) across all contracted and expanded conditions. While there was strong evidence for a change in visual point of subjective normality (PSN, dark grey) due to adaptation, our Bayes factor (BF) analysis provides moderate evidence that adaptation has no effect on tactile distance estimation (TDE, light grey); (BF PSN $=7.73 x$ $\left.10^{10}, B F T D E=0.209\right)$. Grey dots represent individual data. Error bars represent $95 \%$ confidence intervals. 


\section{Discussion}

We were able to replicate the pattern for body size after-effects in the visual domain (Brooks et al., 2016). We found a significant difference for the visual PSN between adaptation for contracted and expanded bodies, with an average shift of PSN in the negative direction for contracted and in the positive direction for expanded body adaptation stimuli, relative to baseline. Replicating a previous finding (Brooks et al., 2016), we also found that adaptation effects were larger for congruent identity (i.e., adapting to self-images and testing with self-images) than for incongruent identity (i.e., adapting to other-images and testing with self-images) conditions.

In contrast, we did not find evidence for visual adaptation effects on tactile distance perception. Neither adaptation to contracted or expanded other-body stimuli, nor of selfbody stimuli, resulted in significant adaptation effects on TDEs in the expected (or opposite) directions. Furthermore, a Bayesian analysis on the data pooled across conditions provides strong evidence for an effect of visual body size adaptation for the visual task and moderate evidence that there is no effect due to adaptation in the tactile task.

On the basis of these analyses, we conclude that visual body size adaptation effects do not transfer to somatosensory body size representations. It is possible that for a manipulation of visual body information to have an effect on somatosensory representations the body has to be viewed in a certain context that promotes associations between the visual stimulus and somatosensory processing of one's own body. Context factors that could matter are the size of the stimuli (our stimuli were smaller than the actual body in contrast to previous work using a life-like stimulus size) and visual perspective (our stimuli were viewed from a second person perspective in contrast to previous work using a first-person perspective) 
(Taylor-Clarke et al., 2004). Furthermore, in the study by Taylor-Clarke et al. (2004)

participants viewed a video-projection of their own hand. Although it was kept relatively still, it is possible that additional synchronous stimulation (synchronous movement or touch) further helped to link visual and somatosensory bodily signals.

Indeed an effect of the synchrony of touch on object size estimations has been shown in a rubber hand illusion study (Bruno \& Bertamini, 2010). In this study, participants viewed artificial hands that were either larger or smaller than their own hand, which was hidden from view. Both hands were then stroked either synchronously or asynchronously, and the experiment tested the effect of these conditions on the haptic object size perception of the size of small disks. They found a significant interaction between the hand and synchrony conditions with larger object size estimates for the larger hand compared to the smaller hand after the synchronous, but not after the asynchronous condition. This suggests that the synchrony of multisensory information contributes to an internal body model that is used as a spatial reference for haptic size perception.

These findings are generally in line with numerous studies using bodily illusions that have shown that specific contexts that promote the association of visual and somatosensory spatial information, such as visual perspective, mirrors and multisensory stimulation, can increase embodiment and spatial interactions between vision and touch (Zopf, Savage, \& Williams, 2010; Maravita, Spence, Sergent, \& Driver, 2002; Petkova, Khoshnevis, \& Ehrsson, 2011; Banakou, Groten, \& Slater, 2013; Pavani, Spence, \& Driver, 2000; Aspell, Lenggenhager, \& Blanke, 2009). In future research it will be important to test whether such conditions would also lead to potential transfer of adaptation effects between modalities. Importantly, as far as we know previous effects of visual signals on tactile distance 
estimation have only been shown for hands and arms. It would thus be important to conduct more studies on other parts of the body, especially body parts people typically have less visual exposure to and are important for body image disturbance, such as the middle part of the body.

Our findings cast doubt on the idea that media exposure of contracted bodies could be a mechanism explaining the findings with respect to tactile distance estimation in Anorexia Nervosa (Keizer et al., 2011; Keizer et al., 2012; Spitoni et al., 2015). Although our findings do not provide evidence against the hypothesis that visual adaptation may contribute to body size misperception in eating disorders and other instances of body image distortions, our results do suggest that at alternative explanations for the findings in the somatosensory domain are needed. For example, it is possible that individuals with eating disorders are relatively more reliant on distorted somatosensory representations (Longo, 2015), potentially due to a change in how spatial visual and somatosensory bodily signals are integrated (Zopf, Contini, Fowler, Mondraty, \& Williams, 2016). More research is needed to understand the role of changes in visual and somatosensory spatial body representations and their integration to further understand body misperceptions in clinical disorders.

In conclusion, we replicated the effects of visual body size adaptation, which leads to significant misperceptions of one's visual body size. We also confirmed that these effects are larger after adaptation to one's own body compared to another body. In contrast, our study provides evidence that this effect does not transfer to misperceiving the spatial distance between two touches on the abdomen. Future research could test whether promoting the link between visual and somatosensory body signals either via mirrors, first- 
person perspective, or multisensory stimulation might lead to a transfer of visual adaptation effects across different modalities.

\section{Acknowledgements}

This project was funded by the ARC Centre of Excellence in Cognition and its Disorders (CCD) Cross Program Support Scheme. RZ was supported by a Discovery Early Career Research Award from the ARC (DE140100499). We thank Jordan Rogers for help with data collection.

Our data and analysis scripts can be found using this Open Science Framework (OSF) link: https://osf.io/ad2yz/?view only=3a829664f13e44e7a4c175c7eeb48f0a.

\section{References}

Aspell, J. E., Lenggenhager, B., \& Blanke, O. (2009). Keeping in touch with one's self: multisensory mechanisms of self-consciousness. PloS One, 4(8), e6488. doi:10.1371/journal.pone.0006488

Banakou, D., Groten, R., \& Slater, M. (2013). Illusory ownership of a virtual child body causes overestimation of object sizes and implicit attitude changes. Proceedings of the National Academy of Sciences of the United States of America, 110(31), 12846-12851.

doi:10.1073/pnas.1306779110 
Brainard, D. H. (1997). The Psychophysics Toolbox. Spatial Vision, 10(4), 433-436.

doi:10.1163/156856897X00357

Brooks, K. R., Mond, J. M., Stevenson, R. J., \& Stephen, I. D. (2016). Body Image Distortion and Exposure to Extreme Body Types: Contingent Adaptation and Cross Adaptation for Self and Other. Frontiers in Neuroscience, 10, 334. doi:10.3389/fnins.2016.00334

Brooks, K. R., Clifford, C. W. G., Stevenson, R. J., Mond, J., \& Stephen, I. D. (2018). The highlevel basis of body adaptation. Royal Society Open Science, 5(6), 172103.

doi:10.1098/rsos.172103

Brooks, K. R., Baldry, E., Mond, J., Stevenson, R. J., Mitchison, D., \& Stephen, I. D. (2019).

Gender and the Body Size Aftereffect: Implications for Neural Processing. Frontiers in Neuroscience, 13, 1100. doi:10.3389/fnins.2019.01100

Brooks, K. R., Mond, J., Mitchison, D., Stevenson, R. J., Challinor, K. L., \& Stephen, I. D. (2019). Looking at the Figures: Visual Adaptation as a Mechanism for Body-Size and -Shape Misperception. Perspectives on Psychological Science, 1745691619869331.

doi:10.1177/1745691619869331

Bruch, H. (1962). Perceptual and conceptual disturbances in Anorexia Nervosa. Psychosomatic Medicine, 2, 187-194.

Bruno, N., \& Bertamini, M. (2010). Haptic perception after a change in hand size. Neuropsychologia, 48(6), 1853-1856. doi:10.1016/j.neuropsychologia.2010.01.006 
Challinor, K. L., Mond, J., Stephen, I. D., Mitchison, D., Stevenson, R. J., Hay, P., \& Brooks, K. R. (2017). Body size and shape misperception and visual adaptation: An overview of an emerging research paradigm. Journal of International Medical Research, 45(6), 2001-2008. doi:10.1177/0300060517726440

de Vignemont, F., Ehrsson, H. H., \& Haggard, P. (2005). Bodily illusions modulate tactile perception. Current Biology, 15(14), 1286-1290. doi:10.1016/j.cub.2005.06.067

Dienes, Z. (2014). Using Bayes to get the most out of non-significant results. Frontiers in Psychology, 5. doi:10.3389/fpsyg.2014.00781

Ernst, M. O., \& Banks, M. S. (2002). Humans integrate visual and haptic information in a statistically optimal fashion. Nature, 415(6870), 429-433. doi:10.1038/415429a

Gadsby, S. (2017). Distorted body representations in anorexia nervosa. Consciousness and Cognition, 51, 17-33. doi:10.1016/j.concog.2017.02.015

Gould-Fensom, L., Tan, C. B. Y., Brooks, K. R., Mond, J., Stevenson, R. J., \& Stephen, I. D. (2019). The Thin White Line: Adaptation Suggests a Common Neural Mechanism for Judgments of Asian and Caucasian Body Size. Frontiers in Psychology, 10, 2532. doi:10.3389/fpsyg.2019.02532

Hummel, D., Rudolf, A. K., Untch, K. H., Grabhorn, R., \& Mohr, H. M. (2012). Visual adaptation to thin and fat bodies transfers across identity. PloS One, 7(8), e43195. doi:10.1371/journal.pone.0043195 
Jeffreys, H. (1961). The Theory of Probability (3rd ed.). Oxford, England: Oxford University Press.

Keizer, A., Smeets, M. A., Dijkerman, H. C., van den Hout, M., Klugkist, I., van Elburg, A., \& Postma, A. (2011). Tactile body image disturbance in anorexia nervosa. Psychiatry Research, 190(1), 115-120. doi:10.1016/j.psychres.2011.04.031

Keizer, A., Smeets, M. A., Dijkerman, H. C., van Elburg, A., \& Postma, A. (2012). Aberrant somatosensory perception in Anorexia Nervosa. Psychiatry Research, 200(2-3), 530-537. doi:10.1016/j.psychres.2012.05.001

Kleiner, M., Brainard, D., Pelli, D., Ingling, A., Murray, R., \& Broussard, C. (2007). What's new in Psychtoolbox-3. Perception, 36(14), 1-16.

Lee, M. D., \& Wagenmakers, E.-J. (2014). Bayesian cognitive modeling: A practical course. Cambridge: Cambridge University Press.

Longo, M. R., Azanon, E., \& Haggard, P. (2010). More than skin deep: body representation beyond primary somatosensory cortex. Neuropsychologia, 48(3), 655-668. doi:10.1016/j.neuropsychologia.2009.08.022

Longo, M. R. (2015). Implicit and Explicit Body Representations. European Psychologist, 20(1), 6-15. doi:10.1027/1016-9040/a000198 
Maravita, A., Spence, C., Sergent, C., \& Driver, J. (2002). Seeing your own touched hands in a mirror modulates cross-modal interactions. Psychological Science, 13(4), 350-355. doi:10.1111/j.0956-7976.2002.00463.x

Medina, J., \& Coslett, H. B. (2010). From maps to form to space: touch and the body schema. Neuropsychologia, 48(3), 645-654. doi:10.1016/j.neuropsychologia.2009.08.017

Mohr, H. M., Rickmeyer, C., Hummel, D., Ernst, M., \& Grabhorn, R. (2016). Altered Visual Adaptation to Body Shape in Eating Disorders: Implications for Body Image Distortion. Perception, 45(7), 725-738. doi:10.1177/0301006616633385

Pavani, F., Spence, C., \& Driver, J. (2000). Visual capture of touch: out-of-the-body experiences with rubber gloves. Psychological Science, 11(5), 353-359. doi:10.1111/14679280.00270

Penfield, W., \& Boldrey, E. (1937). Somatic motor and sensory representation in the cerebral cortex of man as studied by electrical stimulation. Brain, 60(4), 389-443.

Petkova, V. I., Khoshnevis, M., \& Ehrsson, H. H. (2011). The perspective matters! Multisensory integration in ego-centric reference frames determines full-body ownership. Frontiers in Psychology, 2, 35. doi:10.3389/fpsyg.2011.00035

Spitoni, G. F., Serino, A., Cotugno, A., Mancini, F., Antonucci, G., \& Pizzamiglio, L. (2015). The two dimensions of the body representation in women suffering from Anorexia Nervosa. Psychiatry Research, 230(2), 181-188. doi:10.1016/j.psychres.2015.08.036 
Stigler, S. M. (1997). Regression towards the mean, historically considered. Statistical Methods in Medical Research, 6, 103-114.

Sturman, D., Stephen, I. D., Mond, J., Stevenson, R. J., \& Brooks, K. R. (2017). Independent aftereffects of fat and muscle: Implications for neural encoding, body space representation, and body image disturbance. Scientific Reports, 7, 40392. doi:10.1038/srep40392

Tajadura-Jimenez, A., Valjamae, A., Toshima, I., Kimura, T., Tsakiris, M., \& Kitagawa, N. (2012). Action sounds recalibrate perceived tactile distance. Current Biology, 22(13), R516517. doi:10.1016/j.cub.2012.04.028

Taylor-Clarke, M., Jacobsen, P., \& Haggard, P. (2004). Keeping the world a constant size: object constancy in human touch. Nature Neuroscience, 7(3), 219-220. doi:doi.org/10.1038/nn1199

Wickham, H. (2016). ggplot2: Elegant graphics for data analysis. New York: Springer-Verlag. Winkler, C., \& Rhodes, G. (2005). Perceptual adaptation affects attractiveness of female bodies. British Journal of Psychology, 96(Pt 2), 141-154. doi:10.1348/000712605X36343

Zopf, R., Savage, G., \& Williams, M. A. (2010). Crossmodal congruency measures of lateral distance effects on the rubber hand illusion. Neuropsychologia, 48(3), 713-725. doi:10.1016/j.neuropsychologia.2009.10.028 
Zopf, R., Contini, E., Fowler, C., Mondraty, N., \& Williams, M. A. (2016). Body distortions in Anorexia Nervosa: Evidence for changed processing of multisensory bodily signals.

Psychiatry Research, 245, 473-481. doi:10.1016/j.psychres.2016.09.003 